\title{
Research on the Standardization of Drug Test Data
}

\author{
Weiqiang Lin ${ }^{1}$, Guojiang $\mathrm{Xu}^{2}$, Xiaomin Guo ${ }^{1}$, Shuiping $\mathrm{Wu}^{2}$, Jun $\mathrm{Xiao}^{1}$, Senlian $\mathrm{Ye}^{2}$ \\ ${ }^{1}$ Guangdong Institute for Drug Control, Guangzhou, China \\ ${ }^{2}$ Guangdong Wisdom Source Information Technology Limited Company, Guangzhou, China
}

\begin{abstract}
In order to solve the problems that test data of the drug control institution is not standardized and low quality, the data exchange and information sharing are realized, the data value is mined and the information level is improved. Method: combined with the business of control institution and information practice, refer to the practice of relevant standard development; carry out the research on standard development work from the content, principle and process. Result and conclusion: this research completes development of the local standard of Guangdong drug test data, which can provide reference for the development of similar standards in future.
\end{abstract}

Keywords.Drug inspection, data, standards, compilation

\section{Introduction}

Standardization is technology support and important foundation that comprehensively promotes the information; and it is one of the six key factors that form national information [1]. "Guiding Opinions on further strengthening information construction of food and drug supervision delivered by China Food and Drug Administration " (China Food and Drug Administration [2013] No.32) clearly put forward: standardization system of food and drug supervision information is the key construction task, and it is the basis that food and drug supervision information resources external link and service, it is an important protection that improves the overall level of food and drug supervision information construction.

In order to strengthen the food and drug supervision information construction, Information Office of China Food and Drug Administration published, "Food and Drug Supervision Information Standard System" in February 2014 [2], Food and Drug Administration in Guangdong Province and some provinces have released data standards system for drug supervision field inside the province [3]. However, these standards are primarily based on data on drug supervision work and cannot meet the requirements of drug test information for data standards.

In 1996, the Guangdong Provincial Institute for Drug Control independently developed the first set of Windows-based drug testing business management system, and in the province to promote the use of municipal drug testing; 2007, Guangdong Province, the drug testing in the domestic drug testing institutions First
Applied Laboratory Information Management System (LIMS). After more than 20 years of development, Guangdong province's drug testing system in the use of information technology to improve the quality and efficiency of the test at the same time accumulated a large amount of test data, but since the current domestic drug testing data has not yet established standards, the data generated by various agencies is not unified, the data content is not standardized, low data quality, it is difficult to achieve effective data sharing and large data analysis. Drug testing agencies in other provinces in the country are also facing the same problem.

Therefore, planning, development, application and management requirement based on drug control information resources, combined with scientific standardization methods, drug control data standard research and preparation is carried out, improve the standardized system of drug data, strengthen the information work of drug control, achieve drug control data exchange and information sharing, improve the groundwork for of utilization level of drug control data, it has the importance and urgency. This paper integrates practice of local standard research and compiling work of drug test data in Guangdong Province, the content, principle and process of standard for drug test data are discussed.

\section{Compilation Of Content}

\subsection{Standardized Framework}


The standardized framework is the general description of the standard preparation of the entire drug test data, including the composition of standard, logical structure, the sub-item description of the standard system. The standard preparation of drug test data difficult and complex system engineering, which is a top-down normative formulation process. The research and implementation of data standards must be based on a certain standard and standardized framework, and use scientific methods guide it.

"Food and Drug Supervision Information Standard System" includes the overall standards, network infrastructure standards, information resources standards, application support standards, application standards, information security standards, information management standards seven parts. Because the drug test information is closer to drug supervision in the network infrastructure, application support, application, information security and other aspects, so the corresponding drug supervision information standards can be used. Therefore, based on information standard system framework, this topic mainly studies what are directly related to drug test data and the part which is different from drug supervision information standards, the standard of drug test data includes the standard framework, business data standard, data interface standard, standard management method.

\subsection{Business Data Standard}

The business data standard is the core content of the data standard preparation work; it is the standard that verifies business process data of various drugs, the business process includes the whole process of business commission, sample acceptance, sample inspection, report review and report release, the business types include sampling inspection, registration inspection, import inspection, approval of biological products, commissioning, etc..

Business data standard is by analyzing drug test information system needs and scope, the business data preparation terminology and standard standards involved in the drug test industry, data standard, data classification and coding standards, database design specifications. The elaboration scope of basic data information items include the internal identifier, the data element identifier, the data element name, the definition, the data type of the data element value, the representation format, the permission value of data element and so on.

\subsection{Data Interface Standard}

The drug test information system is not an isolated system, in addition to internal management data of drug test; it also involves data exchange among drug test agencies, drug supervision and other departments. Therefore, the drug test data interface does not only include the city institute for drug control, as well as higher-level drug supervision authorities, port drug administration and other institutions. The data interface standard mainly includes the interface specification, the execution order, and dependency relation and so on.

\subsection{Management Method of Standard}

The data standard content of drug test is complex, wide range, long time span and high requirement, it is necessary to standardize the procedure of compiling, implementation and revision, then ensure the formality of the preparation process, the authority of the prepared product, the feasibility of the standard implementation, and normalization of standard revision. Standard management method is authoritatively describe the standard planning, preparation, approval, release, record, implementation, revision and other stages, and data management, data permissions, data storage and other aspects are carried out standardized management.

\section{Compilation Principles}

\subsection{Principle of Authority}

The compiled data standards, first of all, must ensure its authority, namely, the content must comply with relevant laws and regulations of drug supervision, drug test and laboratory management, and the form should meet the requirements of standardization. Business rules and regulations are the guidelines that are jointly followed by the inspection institution, the data standards conform to these laws and regulations, in addition to authority, it more tally with the actual situation of the inspection institutions and reduce the implementation difficulty.

In addition, the rules and regulations are sorted out in accordance with the authority, application scope, the issued departments, and solve the inconsistent problems of different laws and regulations, norms. Some laws and regulations, norms have been improved and refined in the implementation process, the preparation of data standards should also be used as a basis. Data standard preparation work should also develop their own norms, namely, criterion of standard preparation work, avoid similar problems uneven or contradictory, so as to ensure the authority of the standard.

\subsection{Principle of Practicality}

The carrier of information technology standard is the document, if the practicality is poor, and it ultimately will become a pile of waste paper locked in the filing cabinet. The development of data standards is absolutely not a simple standardized professional work. If only the standardized technical personnel carry out data collection, document retrieval, document filing, it will become the mechanical handing of text, no matter what the framework, logical relations, content of data standards are out of line with business, and it ultimately cannot be implemented. Therefore, there must be staff who are familiar with the business fully participate in the preparation of data standards, standardized technical staff 
should also fully understand the business, the relevant regulations and norms of business are regard as the yardstick to meet the construction requirements for management system of drug test. At the same time, we must also fully consider the applied practice of existing information systems and historical data status, and follow the standards and norms of information technology.

Drug test data standards involve a large number of laws, standards and norms, its range is wide and field is broad, overall planning are carried out in accordance with the ontology, system analysis methods, project management and other methods in the standard preparation, these standards and norms are teased out, analyze the internal link its test data is analyzed to ensure the scientific nature, systematic nature, practical applicability universality of standard preparation.

\subsection{Principle of Traceability}

Standard compilation process takes " from inside to outside, bottom up" advance gradually. "From the bottom up", namely start from existing business applications the provincial institute for drug control, the internal management system documents are combed, refer to the existing standards, verify the relevant laws and regulations; "from inside to outside" start from the provincial institute for drug control, representative city institute for drug control are surveyed step by step, and refer to the situation of other provincial institute for drug control. In this process, the definition of data element is cross-referenced, constantly changing, and constantly strive for perfection. Because this work is long span, the number of participants is many, the data is large amount, the process is complex, need technology means to protect the data element of data standard, with the continuous progress of work, the evolution process of data element definition and other contents are effectively recorded, the process changes can be traced, and can back to the source. So the authority and practical applicability of preparation standard can be better ensured.

\section{Compilation Process}

Construction of a set of highly operational, forwardlooking standards must follow a certain process and methods, in-depth research and research, the standard preparation is used to carry out top design, norms, and guide opening and share of drug test information resources, and ultimately achieve scientific applications of data. The process of data standard preparation includes three stages: business research stage, standard preparation stage, review and report stage, the specific process is shown in Figure 1[7].

\subsection{Business Research Stage}

The business research stage mainly use document retrieval, investigation of drug test institution on the spot, business expert' consultation collect the relevant business rules, specifications and software system tables, report forms, interfaces, databases, and extract the corresponding data fields, then analyze and arrange the formed data sets and code sets, the final data element, data element range code will be obtained from these field information, the standard situation of drug test data, user requirements and technical requirements should comprehensively be analyzed, the scope and target of the standard, the construction idea of the conceptual model, and classification research method of the related information resource should be cleared [8].

\subsection{Standard Compilation Stage}

This phase first the actuality of drug test field, according to information theory of conceptual model, information model and data model to analyze systematic function model and application framework, then determine the standard research methods of drug test data. Then, through a series of analysis of process, table, data flow, interface, etc., then develop standardized business function and data resource design framework. Finally, the collected information in business research phase is used, research methods which is identified in earlier stage is utilized, according to the proposed framework, the specific content of data standards of drug test, eventually it is converted to data standard document in line with the standard.

In this process, through the application combing of drug test business, the relevant business applications are sorted out, and tease out existing business information systems (especially the laboratory information management system) business and related forms, sort out the data element, data dictionary; and check the data element and data dictionary by reference to management system documentation within the organization, and study relevant laws and regulations, management norms, calibrate related data and data dictionary, and find the authoritative definition of relevant data element; It is more important to repeatedly communicate and confirm with professional business sector experts. In the field of information technology, according to the relevant standards and norms of information technology and resources, comb the data element data sets, databases and other standard suitable for the test data standards, the overall compilation ideas is shown in Figure 2.

\subsection{Standard Review and Report Stage}

The compilation of standard is a spiral iterative process. After the team forms the standard document, the team should invite the relevant experts in the field of drug test and the experts in the standard to carry out the periodical standard review, special subject review, mid-term review and final report review, and constantly adjust and improve the standard document in accordance with the expert's opinion. The review mainly includes internal self-examination and external expert review.

After the above process completes the compilation of drug test data standards, it can be used as internal 
standards, also further declare it to quality and technology supervision departments as national, local or group standards.

\section{Conclusion}

This research through the combing and analysis of drug test business, combining the current situation of drug test institution information, the compiled content of drug test data standard are summarized from the system framework, data standards, interface standards and management method four aspects. According to the specification of standard preparation, combining the reality of drug test institution, the principle of authority, practicality and traceability should be followed in the standard preparation of drug test data, and analyze the standard preparation process and the work methods and the problems that should be paid attention to. The scientific, systematic and advanced data standards of prepare ideas and methods in this research is used to complete the first domestic drug test data standardpreparation of Guangdong province pharmaceutical inspection data local standard, and it can provide reference for the next similar type of standard preparation.

\section{Acknowledgements}

Fund project: special fund project for public interest research and capacity building in Guangdong province (2014B010103005).

\section{References}

1. The information leadership group office of the Ministry of Water Resources, et al. Information Guidance for Water Conservancy [M], Beijing: China Water\&Power Press, 2003

2. CFDAB/T 0101-2014, Information Standard System of Food and Drug Administration [S]

3. Q/GDYJ 5001-2007, Data Maintenance and Management Standard of Guangdong Food and Drug Administration Industry Standard [S]

4. Lin Weiqiang. Use Computer Technology to Strengthen the Business Management of Institute for Drug Control [J]. Chinese Pharmaceutical Affairs, 1998, 12(2):89-90

5. Lin Weiqiang. Application of LIMS in Guangdong Province Institute for Drug Control [J], Pharmacy Today, 2008, 18(4):88-90

6. Hu Bing, Huang Xiaoqin, Sun Jiaming, Xu Kuidong, Zhang Fan. Research on the Framework of Shanghai Sanitary Supervision Data Standard [J], Chinese Journal of Health Inspection, 2010, 17(3):225-230

7. $\mathrm{Xu}$ Zhan, Wang Hezhen, Chen Jiangling. Research on the Standardization Line of High-End New Electronic Information Industry in Guangdong Province [J], Standard Science, 2013, (04):59-61

8. Hu Bing, Huang Xiaoqin, Feng Donglei, Chang Bo, Sun Jiamin. Research on Developing Method of Shanghai Sanitary Supervision Information Data Standard [J], Computer Applications and Software, 2010, 27(12):299-301 


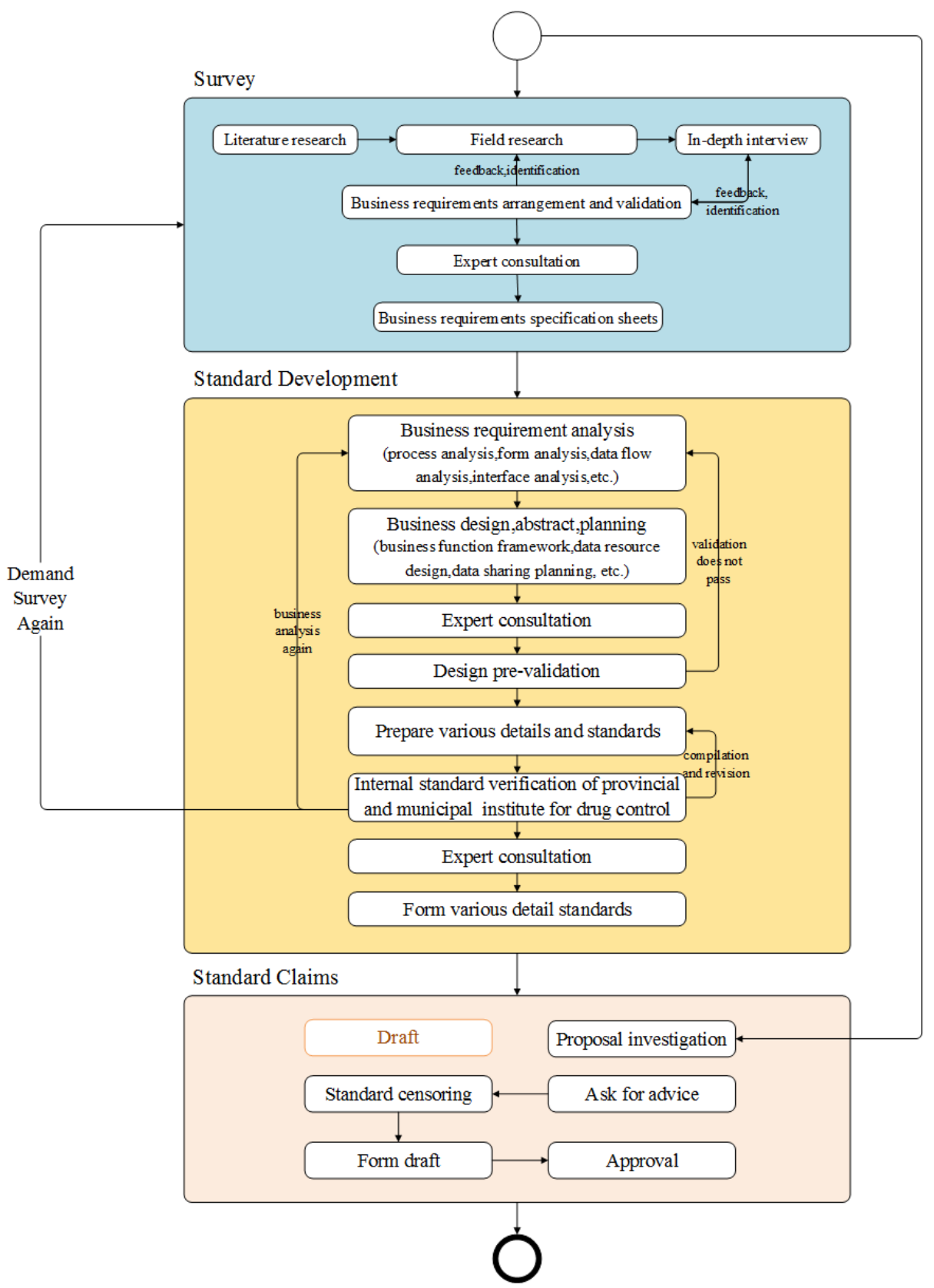

Figure 1. Compilation process of data standard of drug test

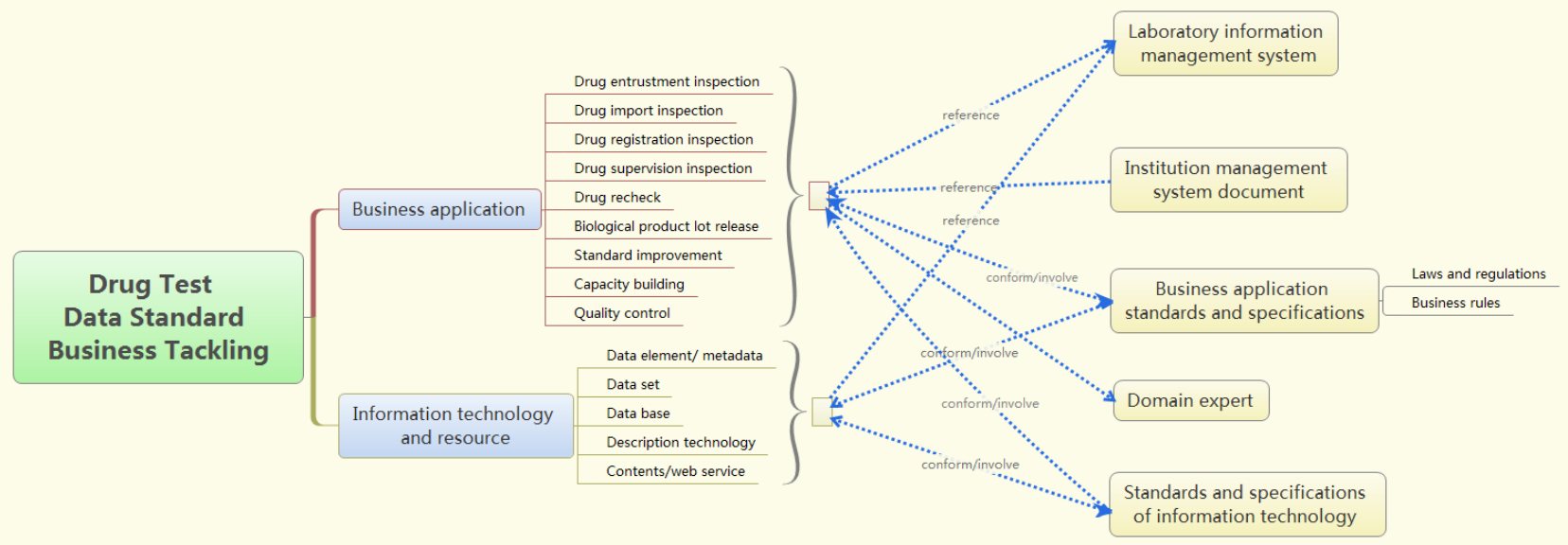

Figure 2. Framework diagram of business tackling of data standard of drug test 